\title{
Steroids control paradoxical worsening of Mycobacterium ulcerans infection following initiation of antibiotic therapy
}

\section{Janine M Trevillyan \\ MB BS, FRACP, \\ Infectious Diseases \\ Physician \\ Paul D R Johnson \\ MB BS, PhD, FRACP, \\ Deputy Director, and Director \\ 1 Department of Infectious Diseases, Austin Health Melbourne, VIC. \\ 2 WHO Collaborating Centre for Mycobacterium ulcerans (Western Pacific Region), Victorian Region), Victorian \\ Reference Laboratory, Melbourne, VIC. \\ janine.trevillyan@ monash.edu}

doi: 10.5694/mjal2.11559

Research p 436

\section{Clinical record}

A 19-year-old man with a 3-month history of a non-healing ulcer of the right knee and indurated oedema involving the entire lower leg (Box 1) presented after not responding to multiple courses of antibiotics. A punch biopsy showed necrosis of the dermis and subcutaneous tissues, and extensive infiltration with extracellular acid-fast bacilli on auramine-rhodamine staining. Polymerase chain reaction (PCR) testing for the IS2404 insertion sequence of Mycobacterium ulcerans was positive, ${ }^{1}$ and $M$. ulcerans was isolated from culture at 6 weeks. The patient reported holidaying on the Bellarine Peninsula, an endemic area for M. ulcerans, 6 months before the development of the lesion. Surgical intervention was deferred due to the extensive area involved and World Health Organization guidelines suggesting antibiotics as first-line treatment for extensive oedematous disease. ${ }^{2}$ He commenced oral daily rifampicin $(600 \mathrm{mg})$ and moxifloxacin $(400 \mathrm{mg})$ and, within a week, noted a marked decrease in exudate and improvement in the painful oedematous disease of the lower leg. Therapy was well tolerated and the ulcer remained stable with only one small area of new skin loss lateral to the primary lesion.

Four weeks later, there was a rapid deterioration in clinical condition, with fevers, sweats, anorexia and a recurrence of lower limb oedema and pain. Significant skin breakdown occurred around the ulcer and distally on the leg, with copious serous exudate (Box 2). Full blood examination showed a neutrophilia (neutrophil count, $14.9 \times 10^{9} / \mathrm{L}$ [reference interval, $1.9-8.0 \times 10^{9} \%$ L]) and a raised C-reactive protein level $(69.8 \mathrm{mg} / \mathrm{L}$ [reference interval, $<3 \mathrm{mg} / \mathrm{L}]$ ). Prednisolone $50 \mathrm{mg}$ daily and intravenous piperacillin-tazobactam $4.5 \mathrm{~g}$ three times daily was commenced. An ultrasound of the limb showed extensive liquefaction of the subcutaneous fat, and swabs were PCRpositive for M. ulcerans but culture-negative following prolonged incubation.

Within 12 hours of commencing prednisolone therapy (combined with broad-spectrum antibiotics), the patient became afebrile, with neutrophil and C-reactive protein levels within reference intervals on full blood examination and an improvement in ulcer appearance. He was discharged on a $25 \mathrm{mg}$ maintenance dose of prednisolone while continuing rifampicin and moxifloxacin. Increasing areas of skin loss and exudate developed when prednisolone was decreased to $12.5 \mathrm{mg}$ daily, and thus he underwent a limited debridement of the lateral knee and negative-pressure wound therapy (VAC GranuFoam dressing, KCI Medical), followed by successful split skin grafting. Heat therapy (Bair Hugger, 3M Arizant Healthcare) was used to maintain skin temperature at $39^{\circ} \mathrm{C}$

Prednisolone was continued for 2 weeks postoperatively ( $15 \mathrm{mg}$ daily) and then stopped without further deterioration in the patient's condition. The patient completed a 3-month course of antimycobacterial antibiotics, at which time there was no sign of ongoing infection. Two skin sites subsequently discharged a small amount of culture-negative material but, at 12 months, there was no evidence of microbiological relapse. ycobacterium ulcerans infection is a geographically restricted infection often referred to by its local name as Daintree, Buruli or Bairnsdale ulcer (BU). The disease occurs in sub-Saharan Africa and within discrete regions of Australia, predominantly coastal Victoria and northern Queensland. Significant diagnostic delays, which place patients at risk of more extensive disease, are common. A recent review identified a mean duration of 42 days of symptoms before diagnosis (range, 2-270 days). ${ }^{3}$ These delays often occur when patients present outside endemic areas, a factor affected by the long incubation period of 4-7 months. ${ }^{4}$

$\mathrm{BU}$ is characterised by slowly progressive skin lesions with local necrosis, destruction of lipocytes and surprisingly little systemic inflammation. This unusual pattern of infection is the result of its unique virulence factor mycolactone, an immunomodulatory toxin that induces necrosis of host tissues and can limit initiation of immune responses and the recruitment of inflammatory cells. ${ }^{5}$ Following the increased use of antibiotics in BU, there have been descriptions of worsening clinical condition after an initial response to therapy. ${ }^{6}$ Such deteriorations (referred to as paradoxical reactions) are most common in patients with extensive disease and can take a variety of forms including increased ulcer size, ulceration of previ- ously non-ulcerative papules or the development of new lesions not detectable before antibiotics. ${ }^{7}$ It is particularly important that paradoxical reactions are not misinterpreted as antibiotic failure, as the vast majority of lesions will resolve without a change in antibiotic regimen.

Paradoxical reactions may be the result of antibioticinduced suppression of mycolactone synthesis, leading to decreased mycolactone concentrations and thus a reversal of macrophage and neutrophil dysfunction with renewed immune surveillance and response. ${ }^{7}$ There are many parallels to the paradoxical reactions described with Mycobacterium tuberculosis in HIV co-infected patients who initiate antiretroviral therapy. In that setting, the reaction likely represents an increased inflammatory response secondary to antiretroviral-induced immune reconstitution. As with $M$. tuberculosis, paradoxical reactions to BU commonly occur 4-8 weeks after therapy commences. ${ }^{7}$

In our case, the deterioration 4 weeks into therapy at the time that mycobacterial cultures became sterile indicates that this was not a failure of antibiotics but an unmasked immune phenomenon. Although our patient did receive a short course of intravenous antibiotics at the time of initiation of steroid therapy, the rapidity of his response and lack of identification of a new bacterial 


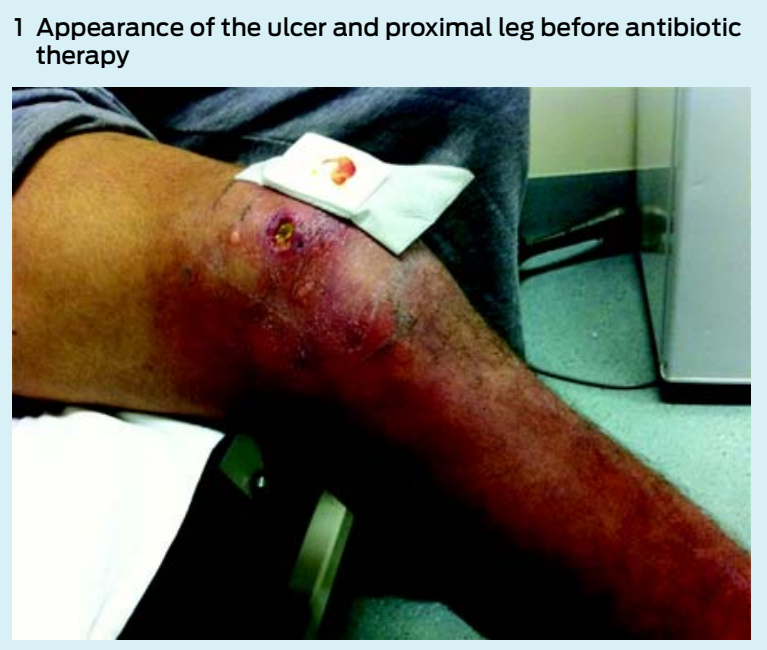

The lesion had been present and undiagnosed for 3 months. Swabs of the ulcer base were positive for acid-fast bacilli (auramine-rhodamine stain) and for Mycobacterium ulcerans: IS2404 polymerase chain reaction test and culture (inoculation on Lowenstein-Jensen media at $30^{\circ} \mathrm{C}$ ).

2 Appearance of the ulcer 4 weeks into antibiotic therapy, at the time of paradoxical reaction

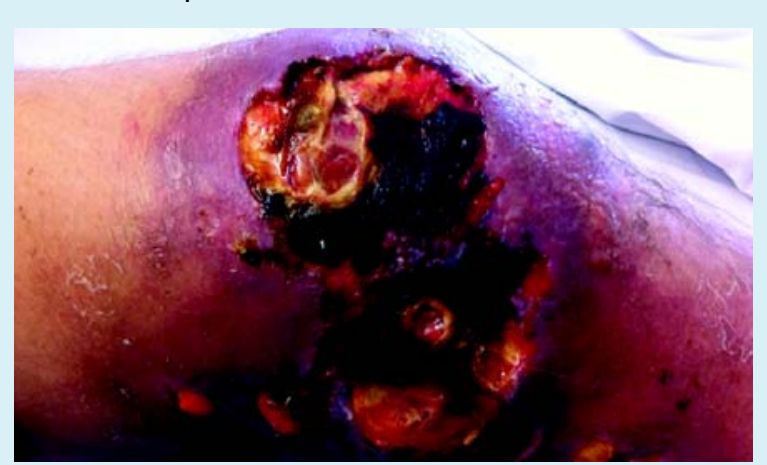

New areas of breakdown were appearing daily with liquefied fatty tissue protruding from the wound and associated with new onset of fevers and a marked increase in pain.

pathogen suggests that the steroid therapy itself was responsible for his improvement. This is supported by the fact that his condition deteriorated when his prednisolone dose was weaned.

It has been shown in animal models that corticosteroids do not adversely affect the outcome of antibiotic therapy in BU, ${ }^{8}$ and Friedman and colleagues reported a marked improvement in the appearance of lesions when steroids were used for paradoxical reactions in this setting. ${ }^{9}$ Established dosing guidelines are available for the management of paradoxical reactions to $M$. tuberculosis (most popularly, $1 \mathrm{mg} / \mathrm{kg} /$ day until improvement, fol-

\section{Lessons from practice}

Mycobacterium ulcerans should be considered in patients who present with non-healing chronic ulcers or atypical cellulitis that do not respond to standard treatment.

- It is common for M. ulcerans infections to worsen following the initiation of antibiotics; these paradoxical reactions are likely due to an enhanced immune response directed at dead and dying bacteria and do not reflect a failure of antibiotic therapy.

- Antibiotics should be continued unchanged when a paradoxical reaction occurs.

- Steroids may play a role in diminishing skin loss and systemic symptoms associated with paradoxical reactions.

lowed by a 2-week wean), ${ }^{10}$ yet the degree of applicability of these guidelines to BU management is unknown.

As treatment evolves from a purely surgical approach to an adjunctive or primary antibiotic focus, optimal management remains controversial. In patients with extensive and oedematous disease, pre-emptive steroid therapy may have a role in preventing paradoxical deteriorations, although to date there is no randomised evidence to support its use in this way.

Acknowledgements: We thank the clinical photography unit at the Austin Hospital and the laboratory staff who performed PCR testing.

Competing interests: No relevant disclosures.

1 Ross BC, Marino L, Oppedisano F, et al. Development of a PCR assay for $r$ apid diagnosis of Mycobacterium ulcerans infection. J Clin Microbiol 1997; 35 : 1696-1700.

2 World Health Organization. Provisional guidance on the role of specific antibiotics in the management of Mycobacterium ulcerans disease (Buruli ulcer). http://www.who.int/buruli/information/antibiotics/en/index.html (accessed Mar 2012).

3 Boyd SC, Athan E, Friedman ND, et al. Epidemiology, clinical features and diagnosis of Mycobacterium ulcerans in an Australian population. Med J Aust 2012; 196: 341-344.

4 Lavender CJ, Fyfe JA, Azuolas J, et al. Risk of Buruli ulcer and detection of Mycobacterium ulcerans in mosquitoes in southeastern Australia. PLoS Negl Trop Dis 2011; 5: el305.

5 Adusumilli S, Mve-Obiang A, Sparer T, et al. Mycobacterium ulcerans toxic macrolide, mycolactone modulates the host immune response and cellular location of M. ulcerans in vitro and in vivo. Cell Microbiol 2005; 7: 1295-1304.

6 O'Brien DP, Robson ME, Callan PP, McDonald AH. "Paradoxical" immunemediated reactions to Mycobacterium ulcerans during antibiotic treatment: a result of treatment success, not failure. Med J Aust 2009; 191: 564-566.

7 Nienhuis WA, Stienstra Y, Abass KM, et al. Paradoxical responses after start of antimicrobial treatment in Mycobacterium ulcerans infection. Clin Infect Dis 2012; 54: 519-526.

8 Martins TG, Trigo G, Fraga AG, et al. Corticosteroid-induced immunosuppression ultimately does not compromise the efficacy of antibiotherapy in murine Mycobacterium ulcerans infection. PLoS Negl Trop Dis 2012; 6: el925.

9 Friedman ND, McDonald AH, Robson ME, O'Brien DP. Corticosteroid use for paradoxical reactions during antibiotic treatment for Mycobacterium ulcerans. PLoS Negl Trop Dis 2012; 6: el767.

10 American Thoracic Society; Centers for Disease Control and Prevention; Infectious Diseases Society of America. Treatment of tuberculosis. MMWR Recomm Rep 2003; 52 (RR-11): 1-77. 